\title{
Die bitteren Lehren von Minamata
}

\section{Rudolf Stumberger}

Dr. phil., freier Journalist

Minamata ist eine kleine Stadt mit rund 25000 Einwohnern an der Westküste von Kyushu, der südlichsten der fünf Hauptinseln von Japan. Der Ort in der Präfektur Kumamoto besteht vor allem aus der langgezogenen Hauptstrasse mit ihren Kaufhäusern und anderen Läden. Unten am Hafen stehen noch immer die alten Fabrikgebäude und nicht weit davon ein moderner Baukomplex mit benachbarter grosser Rasenfläche. Hier in Minamata entstand 1971 ein Foto, das um die Welt ging: Die Mutter Ryoko Uemura badet ihre behinderte Tochter Tomoko in einem traditionellen japanischen Bad. Der Fotograf war Eugene W. Smith, ein Amerikaner, der später von Arbeitern der Fabrik zusammengeschlagen wurde. Tomoko war ein junges Mädchen, das schwere Schäden durch eine Quecksilbervergiftung erlitten hatte. Der Grund dafür waren die giftigen Abwässer, welche die örtliche Chemiefabrik Chisso Corporation jahrelang in das Meer geleitet hatte. Über die Fische gelangte dann das Quecksilber in die Menschen. Heute erinnert in Minamata ein eigenes Museum sowie ein Forschungsinstitut an die Umweltkatastrophe aus den 1960er Jahren, die als «Minamata-Krankheit» bezeichnet wurde.

\section{Seltsame Dinge geschahen}

Vor gut 60 Jahren war das Yatsushiro-Meer um die Bucht von Minamata reich gesegnet mit Fischen aller Art, die für die Fischer der Stadt eine anständige Lebensgrundlage boten. Das meiste, was auf die Essteller der örtlichen Familien kam, war Reis und Fisch, das Nachkriegsjapan dieser Zeit war ein armes Land. Doch in den 1950er Jahren geschahen seltsame Dinge in der Bucht. Die Krebse begannen zu sterben, Fische trieben an der Wasseroberfläche, die Algen wuchsen nicht mehr und Katzen zeigten ein merkwürdiges Verhalten, bevor sie aus mysteriösen Gründen starben. Am 21. April 1961 wurde ein Kind aus Minamata mit ernsthaften Beschwerden in das örtliche Hospital gebracht, es konnte nicht mehr gehen, sprechen und essen. Andere Einwoh-

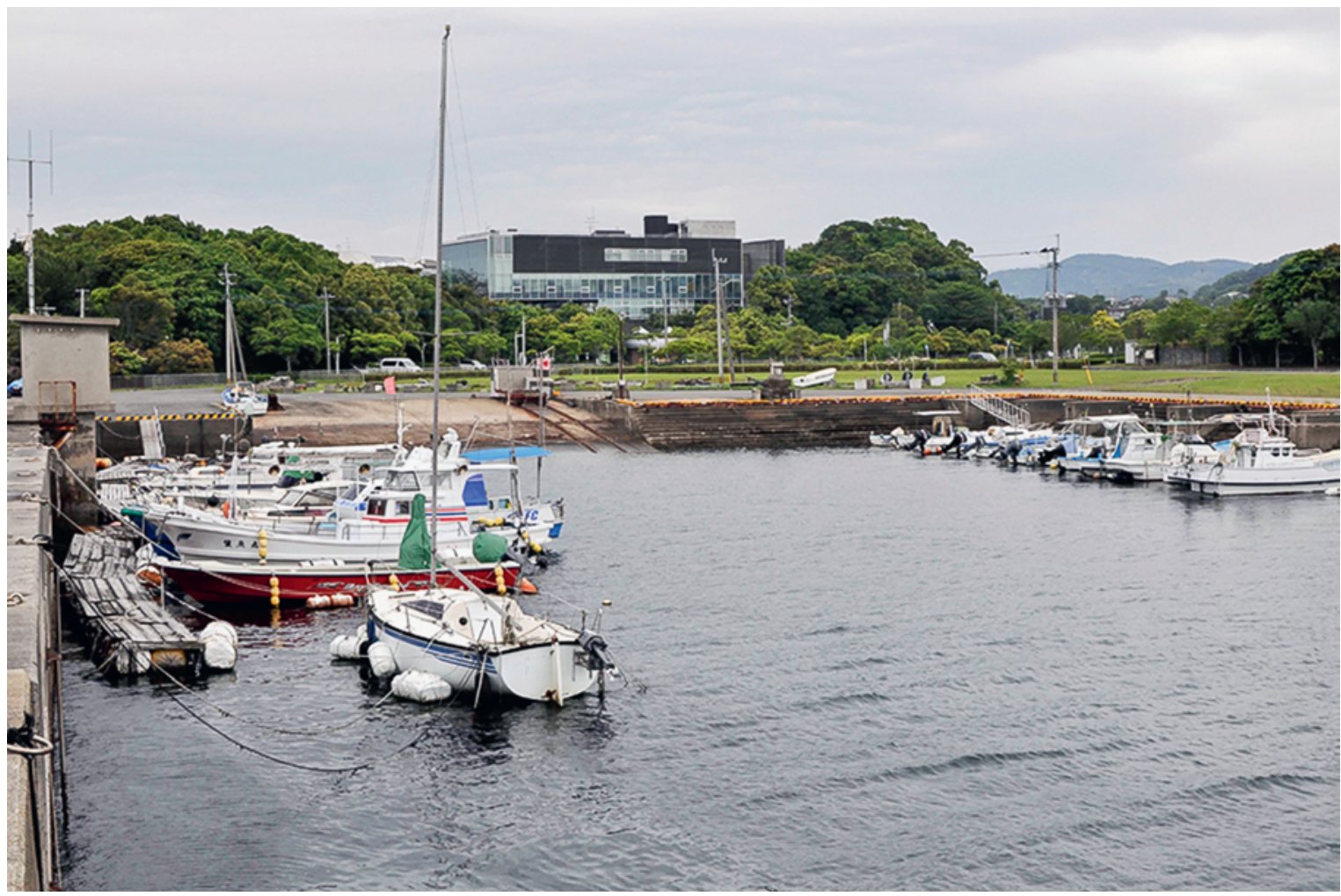

Blick vom Hafen aus auf das Museum und Minamata-Archiv (im Hintergrund). 
ner folgten. Am 1. Mai 1961 unterrichtete der Krankenhausdirektor das Gesundheitsamt, dass sich vier Patienten mit zerebralen Schädigungen in Behandlung befänden, die Ursache sei unbekannt. Das war der Zeitpunkt der offiziellen Wahrnehmung der «Minamata Disease». Sieben Jahre und viele wissenschaftliche und medizinische Untersuchungen später, am 26. September 1968, gab die Regierung ihre offizielle Stellungnahme bekannt. Danach war die «Minamata Disease» eine Schädigung des zentralen Nervensystems, hervorgerufen durch eine Methylquecksilberverbindung. Das Quecksilber stamme aus der örtlichen Chemiefabrik, wo es als ein Nebenprodukt der Herstellung von Acetaldehyd entstand und als Abwasser ungefiltert in das Meer geleitet wurde. Das Quecksilber habe sich in Fischen und anderen Meerestieren angesammelt und führte bei den Einwohnern, die viel von den vergifteten Meeresfrüchten assen, und bei Neugeborenen zu Schädigungen. Insgesamt waren mehr als 2200 Menschen betroffen.

\section{Minamata - eine «Company town»}

Die Geschichte von Minamata ist auch eine Geschichte über den industriellen Aufstieg Japans in den 1950er und 1960er Jahren. Um die Landwirtschaft auf den von Bergen und dem Meer begrenzten Anbauflächen voranzubringen, wurde Kunstdünger genutzt. Und diesen stellte die Chemiefabrik von Minamata her, die Stadt war quasi eine «Company town", in der ein Arbeitgeber die meisten Arbeitsplätze stellte. Die Arbeiter der Fabrik wurden als «Kaisha-yuki san» beneidet, das Unternehmen organisierte Konzerte und Sportfestivals und

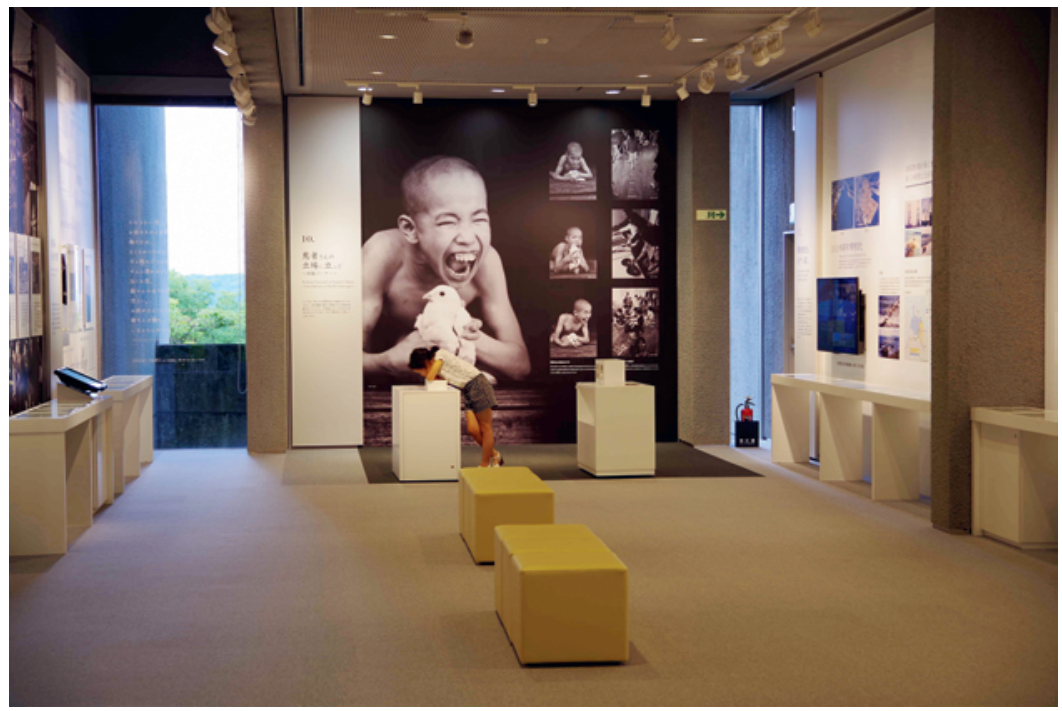

Eine Fotoausstellung im Innern des Museums. lieferte der Stadt mehr als die Hälfte der Steuereinnahmen. Ein Viertel des Stadtgebietes gehörte der Firma. Das Auftauchen der Krankheit führte zur sozialen Spaltung zwischen den Opfern und ihren Familien einerseits und dem Unternehmen und seinen Unterstützern andererseits. Die Opfer kämpften gegen die Macht des Unternehmens, versammelten sich zu Protesten vor den Werkstoren und klagten vor Gericht. Bei Kundgebungen zeigten Angehörige die Bilder der Verstorbenen und trugen Fahnen mit der Aufschrift «Wut». Auf diese aufgeheizte Situation traf der Fotograf Eugene W. Smith, als er 1971 nach Minamata kam, um die ökologische Katastrophe und ihre Opfer zu dokumentieren. Smith war damals 53 Jahre alt, hatte als Kriegsfotograf im Pazifik überlebt und sich einen Namen als sozial engagierter, unabhängiger - und immer in Geldschwierigkeiten steckender - Bildberichterstatter gemacht. Sein Foto der verkrüppelten 15-jährigen Tomoko ging um die Welt. Im Januar 1972 wurde Smith bei einem Angriff von Beschäftigten des Chemieunternehmens so schwer verletzt, dass er jahrelang unter heftigen Schmerzen litt und Selbstmord begehen wollte.

\section{Minamata heute}

Wer heute durch Minamata geht, findet ebenfalls fotografische Hinweise auf die «Minamata Disease». Ein Plakat an einer Hauswand gegenüber dem Polizeirevier weist auf eine Ausstellung im städtischen $\mathrm{Mu}$ seum hin. Dieses Museum wurde 1993 eröffnet und zeigt über vier Abteilungen die Geschichte der ökologischen Katastrophe. In der Eintrittshalle wird der Besucher mit der Region um Minamata vertraut gemacht: Wie die Küstenbewohner als Fischer seit Jahrhunderten mit dem Meer lebten und daraus ihre Nahrung bezogen. Die zweite Abteilung widmet sich der Geschichte der örtlichen Chemiefabrik. 1908 gegründet, wurde die Chisso Corporation zu einem bedeutenden Industrieunternehmen Japans, und der wirtschaftliche Aufschwung kam Minamata zugute, die Stadt wuchs. 1932 war Chisso eines der ersten Unternehmen in Japan, die Acetaldehyd produzierten, das bei der Herstellung von Plastik und anderen Stoffen verwendet wurde. In den 1930er Jahren wurde Chisso schliesslich zu einem führenden Chemie-Unternehmen. Die Ausstellung zeigt die Fabrik in den 1950er Jahren, als über einen Abwasserkanal das Quecksilber ins Meer geleitet wurde, und informiert über die ersten Anzeichen der «Minamata Disease».

Die dritte Abteilung widmet sich den Opfern. Die Quecksilbervergiftung verursachte Sehstörungen, das Hörvermögen war eingeschränkt, ebenso die Koordinationsfähigkeit von Händen und Füssen. Manche 


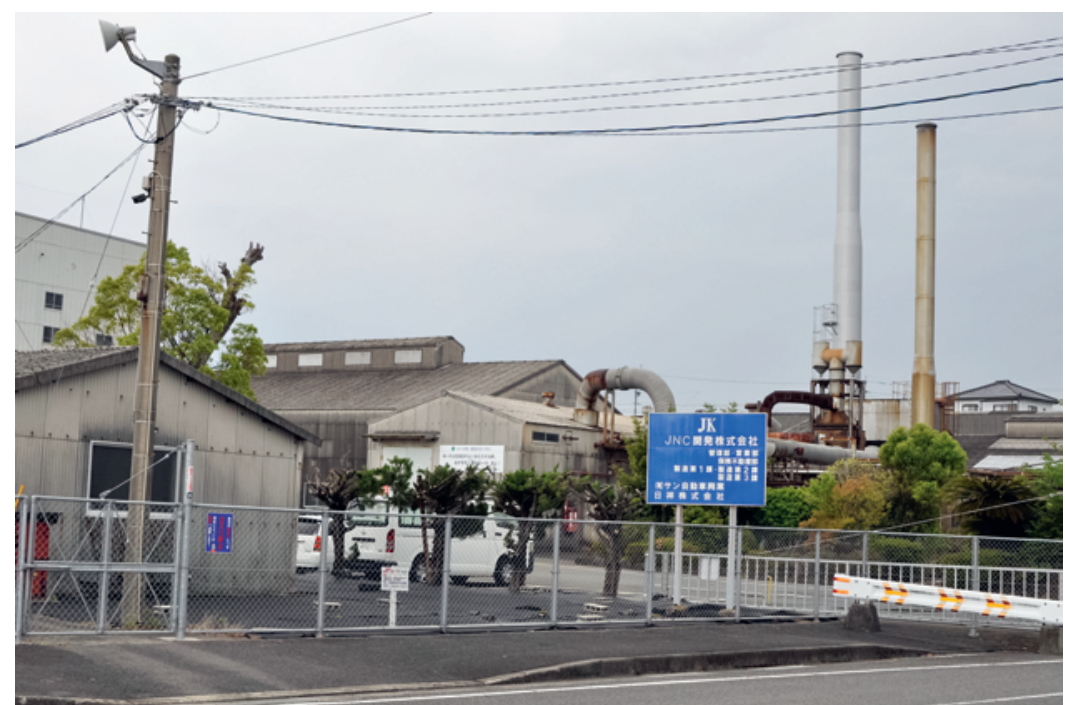

Am Hafen sind noch immer Anlagen der Chemiefabrik zu sehen.

verloren den Geschmackssinn und das Gefühl in den Fingerspitzen, Babys wurden verkrüppelt geboren. Insgesamt wurden bis 2015 in den Präfekturen Kumamoto und Kagoshima 2278 Opfer der Krankheit identifiziert. Die Ausstellung thematisiert auch den Kampf dieser Opfer um ihre Anerkennung und um Entschädigungen durch das Unternehmen und durch den Staat. 2009 wurden Entschädigungen an über 60000 Ange- hörige gezahlt, doch der Minamata-Fall schwelt noch immer und ist noch nicht beendet. Die vierte Abteilung schliesslich zeigt die Lehren, die Minamata aus den Vorfällen gezogen hat. Die Stadt versteht sich heute als ökologische Modellstadt und hat sich den Kampf gegen die Umweltverschmutzung durch Quecksilber auf die Fahnen geschrieben. Im Oktober 2013 wurde das Minamata-Abkommen über die weltweite Reduzierung von Quecksilberemissionen auf den Weg gebracht, nie wieder soll sich eine derartige Katastrophe wiederholen.

Vor dem Museum befindet sich der grasbewachsene Küstenabschnitt, auf dem ab 1980 die Quecksilberablagerungen abgebaut und neu aufgeschüttet wurde. Hier findet sich auch das Denkmal, das an die «Minamata Disease» erinnern soll. In direkter Nachbarschaft zum Museum ist auch das nationale Forschungsinstitut und Archiv zum Thema angesiedelt. Und nur wenige Schritte vom Museumseingang entfernt wurde 1996 ein weiteres Mahnmal errichtet: Es zeigt 108 nichtrostende Stahlkugeln, die über eine schiefe Ebene verteilt sind. Sie symbolisieren Quecksilberkugeln, die in das Meer rollen.

\section{Bildnachweis}

Rudolf Stumberger
Korrespondenz: Dr. Rudolf Stumberger Münchner Pressebüro Fliegenstr. 12/I D-80337 München Stumberger[at]muenchnerpressebuero.de

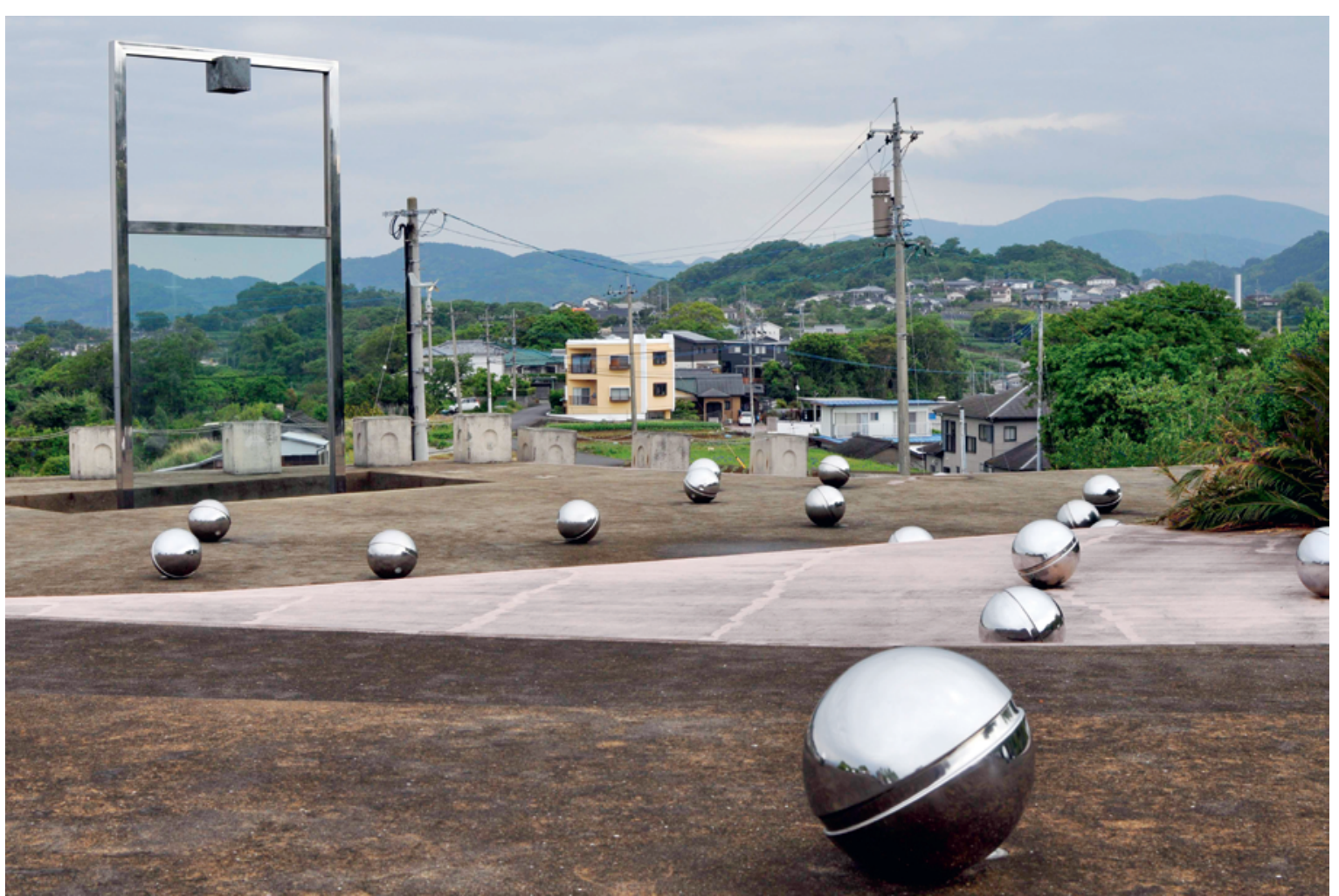

Das Denkmal zur Minamata-Katastrophe: Silberkugeln, die wie Quecksilber in Richtung Meer rollen. 